\title{
Editorial
}

\section{Vitamin D and mental health during the Covid-19 outbreak}

\author{
Séphora Natércia Albuquerque Oliveira' ${ }^{1}$, Modesto Leite Rolim Neto $^{2}$
}

'School of Medicine of Juazeiro do Norte - FMJ/Estácio, Juazeiro do Norte, Ceará, Brazil.

${ }^{2}$ School of Medicine, Federal University of Cariri - UFCA, Barbalha, Brazil

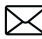

Modesto Leite Rolim Neto School of Medicine

Universidade Federal do Cariri - UFCA.

Phone: (+55 88) 999042979

modesto.neto@ufca.edu.br

Keywords:

Vitamin D

Depressive Disorder;

COVID-19

Social Isolation

Pandemic

\begin{abstract}
The relationship between hypovitaminosis $D$ and depressive disorder is well documented in the medical literature. However, the biological mechanisms by which vitamin D can modulate psychological distress are still unclear. Containment measures can decrease individual's exposure to the sun, significantly increasing their needs for vitamin $D$, a nutrient already deficient in patients with depression. Therefore, it can be inferred that by ingesting the same amount of vitamin $D$, depressive individuals seem to obtain a lesser amount of this nutrient from the sun's rays when compared to healthy people. We found the relationship between vitamin D and COVID-19 has been increasingly studied, mainly due to the changes that this substance can cause in the inflammatory process - especially in the release of cytokines, in SARS and in lung injuries. Despite the benefits, the existing observational studies on this exchange are not enough to definitively associate vitamin D as a protective factor for COVID-19.
\end{abstract}


COVID-19, caused by the SARS-CoV-2 virus, was initially identified in Wuhan, China, and quickly reached several countries, being declared a pandemic by the World Health Organization (WHO) in March 2020. The transmission of this disease occurs through respiratory tract fluids and the symptoms are diverse, including fever and cough. ${ }^{\prime}$ In view of this scenario, with the objective of limiting the spread of the disease, countries have adopted measures of social distancing. However, it's noticeable that the measures had a negative impact on the mental health of individuals, with the risk of developing psychological problems or aggravating existing mental illnesses. These aggravating factors include the fear of contracting the infection, economical loss, and stigmas related to COVID-19. ${ }^{2}$

This drastic change in routine has impacted the mental health of several social groups and may be associated with a feeling of frustration and boredom. In this sense, quantitative studies among quarantined people have shown high rates of symptoms of psychological distress such as stress, insomnia, and depression. ${ }^{2}$ In addition, containment measures can decrease individual's exposure to the sun, significantly increasing their needs for vitamin $D, a$ nutrient already deficient in patients with depression when compared to healthy people of the same age and sex. ${ }^{3,4}$

Vitamin D is a fat-soluble compound found naturally in few foods such as fish with high levels of oil, cod liver oil, egg yolks, shiitake mushrooms, liver, and other entrails. Approximately $90 \%$ of it's absorbed after exposure to ultraviolet-B (UVB) radiation, with dermal synthesis being the main route to obtain it. ${ }^{5}$

The relationship between hypovitaminosis $D$ and depressive disorder is well documented in the medical literature. However, the biological mechanisms by which vitamin $D$ can modulate psychological distress are still unclear and require further investigation. ${ }^{5}$ By ingesting the same amount of vitamin $D$, depressive individuals seem to obtain a lesser amount of this nutrient from the sun's rays when compared to healthy people. However, it has been shown that sun exposure is the main factor involved in the occurrence of vitamin $D$ deficiency for both patients with depression and healthy individuals. ${ }^{3}$ Therefore, it can be inferred that social isolation is closely related to a reduction in serum levels of this substance, as it can decrease people's exposure to sunlight.
In addition, studies have shown that vitamin $D$ has an assisting function in the immune system, especially regarding the modulation of the inflammatory process during viral infection. ${ }^{6}$ In this sense, individuals with vitamin $D$ deficiency are more susceptible to various infectious diseases, with an emphasis on viral infections that affect the upper respiratory tract. ${ }^{7}$ Still in this context, some experimental research suggests the role of vitamin $D$ as a mitigating factor of important lung damage in the pathogenesis of COVID-19, as well as indicating the association between vitamin $D$ and the lower concentration of interleukin-6 (IL-6), a cytokine that significantly influences SARS-CoV-2-induced severe respiratory syndrome (SARS). ${ }^{6,7}$ Although the role of vitamin $D$ in combating COVID-19 is imprecise, it has been shown that supplementation with vitamin D3 is associated with a reduction in the length of hospital stay for patients affected by infectious diseases of the respiratory tract, such as COVID-19.8

Therefore, the negative implications of vitamin $D$ deficiency on mental health are even more evident in the current pandemic context. The need for social isolation reduces the frequency of exposure to solar radiation and thus the ability to obtain this nutrient. Many studies have found the presence of hypovitaminosis $D$ in patients with depressive disorder, a health condition considerably impacted by the pandemic scenario.

Finally, the relationship between vitamin D and COVID-19 has been increasingly studied, mainly due to the changes that this substance can cause in the inflammatory process - especially in the release of cytokines, in SARS and in lung injuries. Despite the benefits, the existing observational studies on this exchange are not enough to definitively associate vitamin $\mathrm{D}$ as a protective factor for COVID-19. Controlled and randomized studies are necessary to test and prove this relationship. Although the protective and therapeutic effect of vitamin D against COVID-19 is not confirmed, there are reasons for recommending supplementation of this nutrient to avoid its deficiency during this pandemic.

\section{Acknowledgment}

The authors would like to thank the Research Group: Suicidology - Universidade Federal do Ceará (UFC) and Brazilian National Council for Scientific and Technological Development (CNPq) - institution linked to the Brazilian Department of Science, Technology and Scientific Writing Lab, Medicine School - Universidade Federal of Cariri (UFCA). 


\section{Funding}

The Research Group: Suicidology - Universidade Federal do Ceará (UFC) and Brazilian National Council for Scientific and Technological Development $(\mathrm{CNPq})$ - institution linked to the Brazilian Department of Science, Technology and Innovation to encourage research in Brazil.

\section{Conflict of interest}

The authors declare that they have no competing interests.

Séphora Natércia Albuquerque Oliveira https://orcid.org/0000-0002-1381-7453 Modesto Leite Rolim Neto https://orcid.org/0000-0001-9379-2120

\section{References}

1. Burhamah $W$, AlKhayyat $A$, Oroszlányová $M$, AlKenane A, Almansouri A, Behbehani M, . . AlSuwaidan M. The psychological burden of the COVID-19 pandemic and associated lockdown measures: Experience from 4000 participants. J Affect Disord 2020;277:977985 Doi:10.1016/i.jad.2020.09.014

2. Brooks SK, Webster RK, Smith LE, Woodland L, Wessely S, Greenberg N and Rubin GJ. The psychological impact of quarantine and how to reduce it: rapid review of the evidence. Lancet 2020;395(10227):912-920 Doi:10.1016/ s0140-6736(20)30460-8
3. Jahrami H, Bragazzi NL, Grant WB, AlFarra HSM, AlFara WSM, Mashalla S and Saif Z. Vitamin D Doses from Solar Ultraviolet and Dietary Intakes in Patients with Depression: Results of a Case-Control Study. Nutrients 2020; 12(9):2587 Doi:10.3390/nu 12092587

4. Meltzer DO, Best TJ, Zhang H, Vokes T, Arora $V$ and Solway J. Association of Vitamin D Status and Other Clinical Characteristics With COVID-19 Test Results. JAMA Netw Open 2020;3(9):e2019722 Doi:10.1001/jamanetworkopen.2020.19722

5. Di Nicola M, Dattoli L, Moccia L, Pepe M, Janiri D, Fiorillo A, . . Sani G. Serum 25-hydroxyvitamin D levels and psychological distress symp patients with affective disorders during the COVID-19 pandemic. Psychoneuroendocrinology 2020; 122: 104869 Doi: 10.1016/i.psyneven. 2020.104869

6. Laird E, Rhodes J and Kenny RA. Vitamin D and Inflammation: Potential Implications for Severity of Covid-19. Ir Med J 2020; $113(5): 81$

7. Mitchell F. Vitamin-D and COVID-19: do deficient risk a poorer outcome? Lancet Diabetes Endocrinol 2020;8(7):570 Doi:10.1016/ s2213-8587(20)30183-2

8. Grant WB, Lahore H, McDonnell SL, Baggerly CA, French CB, Aliano JL and Bhattoa HP. Evidence that Vitamin D Supplementation Could Reduce Risk of Influenza and COVID-19 Infections and Deaths. Nutrients 2020;12(4):988 Doi: 10.3390/nu 12040988 